\title{
Wrong Sign Contamination in NOvA
}

Abhilash Yallappa Dombara (Syracuse University) for NOvA collaboration

Syracuse

University

\section{NOvA experiment}

- NOvA is an off-axis (14.6 mrad), long baseline $(810 \mathrm{~km})$ neutrino oscillation experiment which uses NuMI beam at Fermilab.

- NOvA's main goal is to use muon neutrino disappearance and electron neutrino appearance to probe neutrino mass ordering, $\Delta m_{32}^{2}, \theta_{23}$ and $\delta_{c p}$

- NOvA uses two functionally identical liquid scintillator detectors.

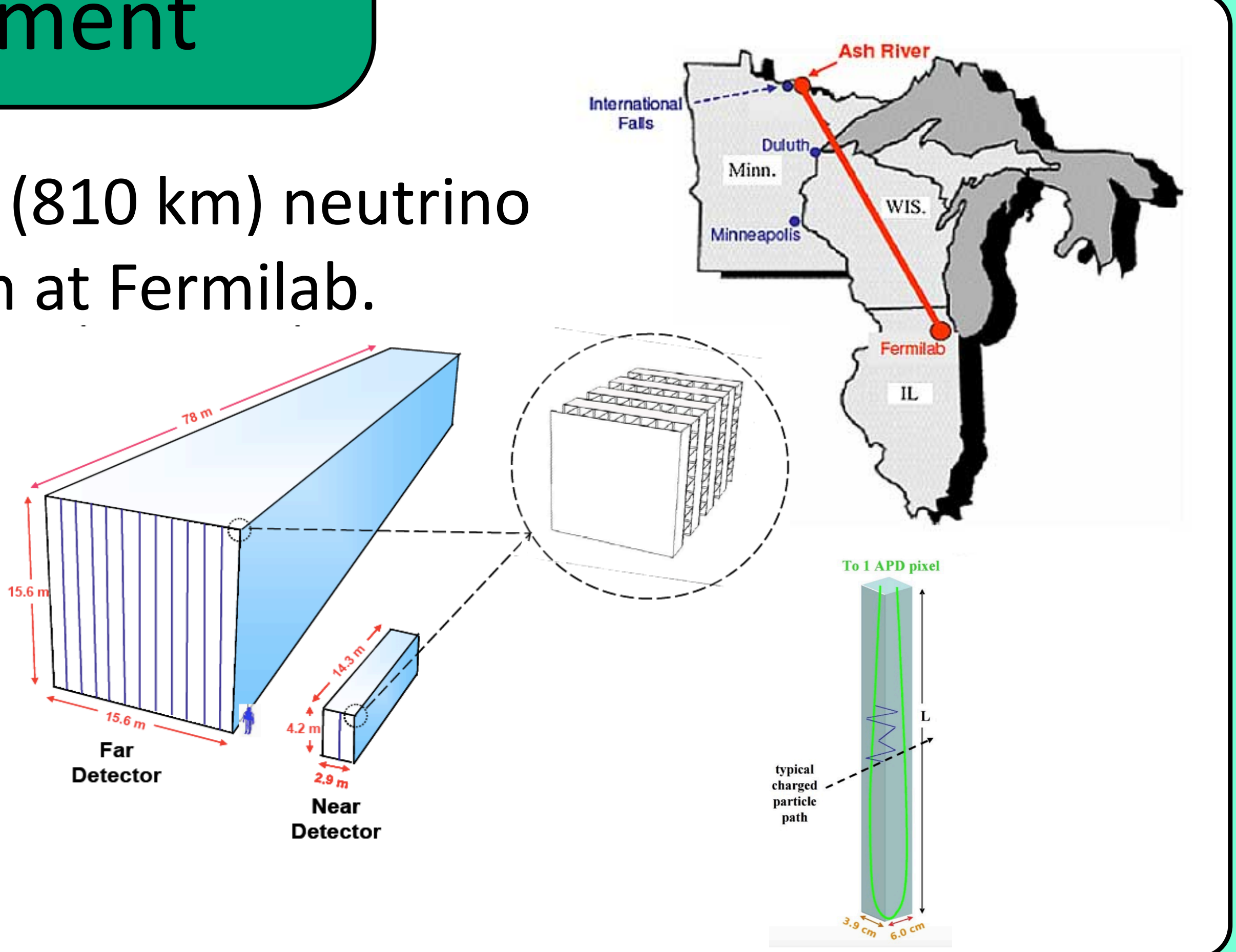

\section{Analysis procedure}

- The wrong sign fraction is estimated for electron neutrinos and muon neutrinos separately.

- CNN (Convolutional Neural Network) was trained on simulation to identify neutrino and antineutrino events and validated with data.

- A optimal cut value was chosen for Wrong Sign CNN ID based on efficiency and purity to separate the whole sample into wrong sign and right sign enhanced regions.

- Compute the wrong sign fraction using matrix method

$\alpha, \beta:$ Fit parameters

D: Data

$\mathrm{S}:$ Signal $=v_{\mathrm{e}}, v_{\mu} \mathrm{CC}$ and NC

B: Background $=\bar{v}_{e}, \bar{\nu}_{\mu} \mathrm{CC}$ and NC

WS: Wrong sign enhanced

$\alpha B_{W S}+\beta S_{W S}=D_{W S}$

$$
\beta\left(S_{R S}+S_{W S}\right)
$$

Wrong sign fraction $=\frac{\beta\left(S_{R S}+S_{W S}\right)}{\beta\left(S_{R S}+S_{W S}\right)+\alpha\left(B_{R S}+B_{W S}\right)}$

Wrong sign error $=\sqrt{\frac{C_{22}\left(S_{R S}+S_{W S}\right)^{2}}{\left(D_{R S}+D_{W S}\right)^{2}}}$

$$
\text { Covariance matrix : } \mathrm{C}=M^{-1}\left[\begin{array}{cc}
\sigma_{D R S} & 0 \\
0 & \sigma_{D W S}
\end{array}\right] M^{T}
$$
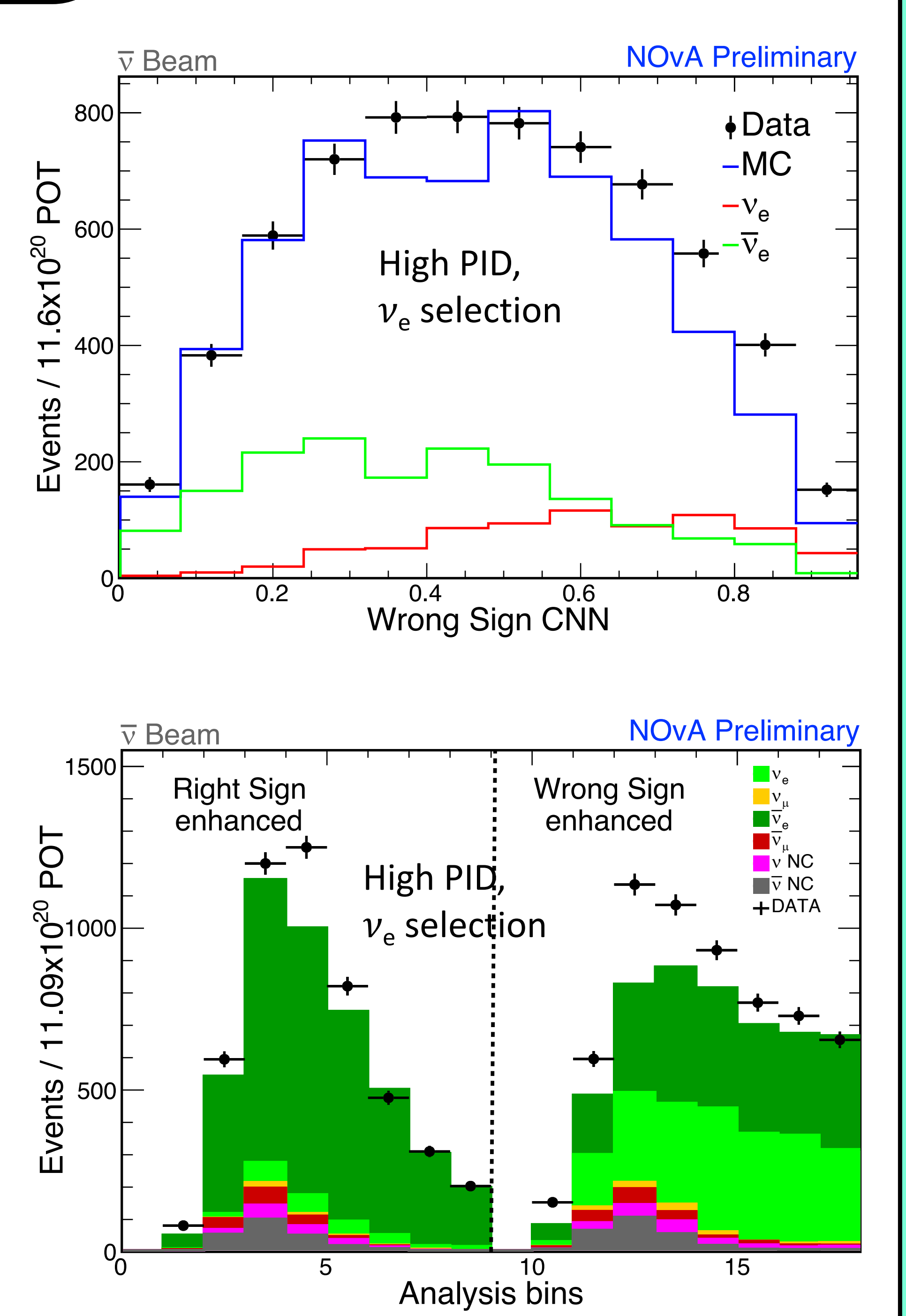

Matrix : $M=\left[\begin{array}{cc}B_{R S} & S_{R S} \\ B_{W S} & S_{W S}\end{array}\right]$

\section{Wrong Sign contamination}

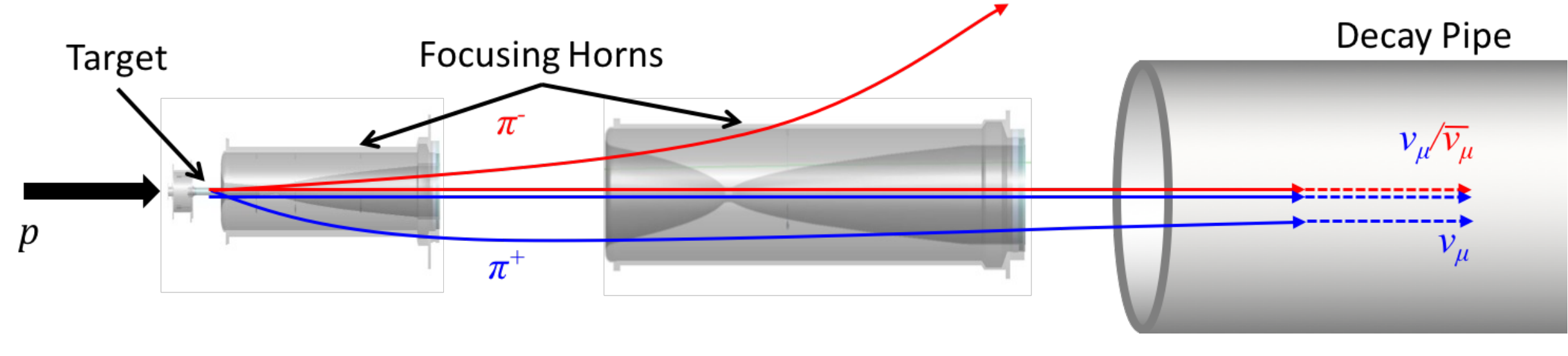

- Wrong sign contamination is fraction of neutrinos/antineutrinos in antineutrino/neutrino beam.

- Wrong sign fraction is higher in antineutrino beam.

- Wrong sign fraction affects our mass hierarchy and $\delta c p$. measurements because these parameters are estimated based on difference between $v_{e}$ and $\bar{v}_{\mathrm{e}}$ appearance.
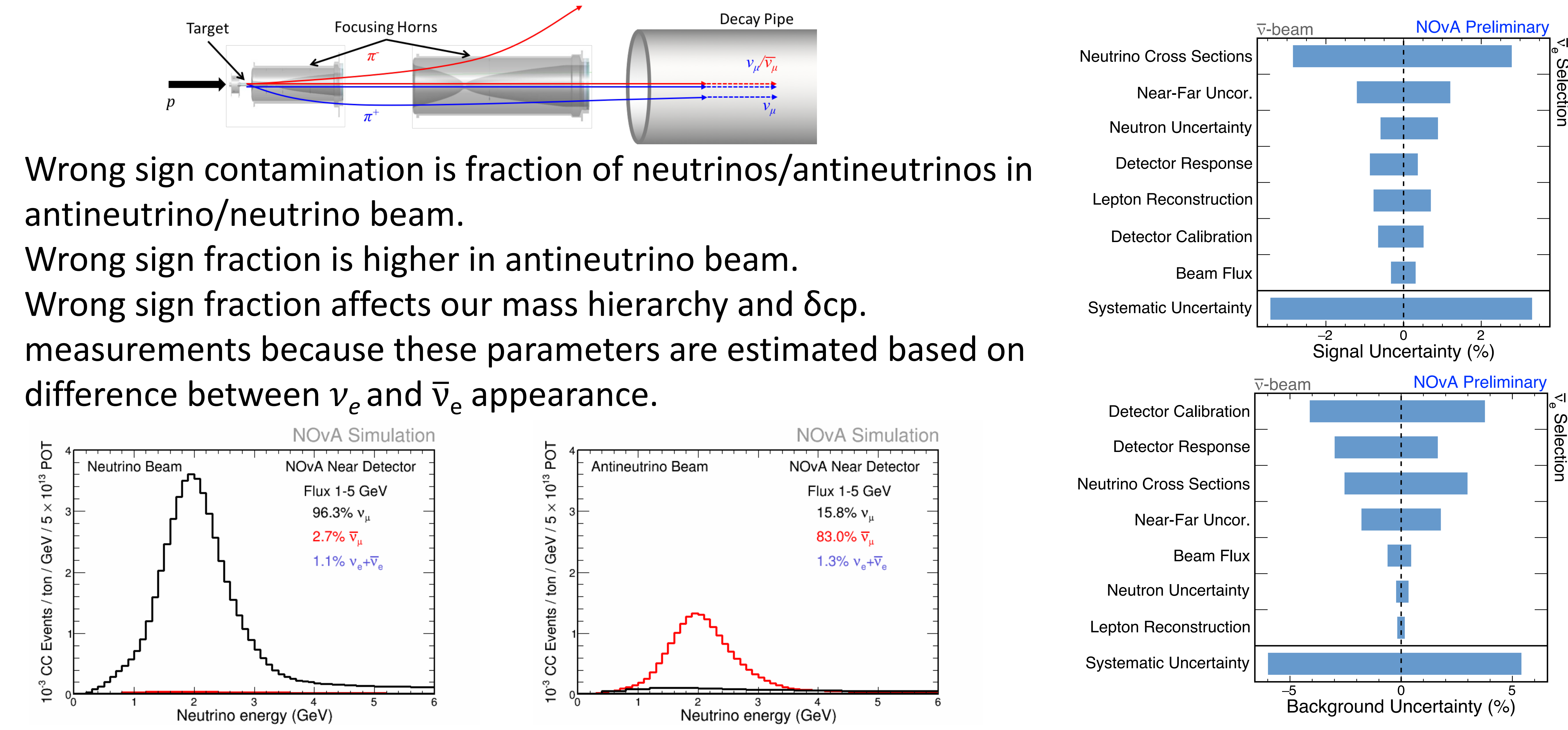

\section{Analysis Methods}

- Neutron capture method: Neutrinos are more likely to produce $\mu^{-}$which gets absorbed in nucleus and produce delayed neutrons while antineutrinos don't.

- Prong CVN score: Identify neutrinos using a single particle classifier for proton identification

- Wrong Sign CNN score: Dedicated CNN for classifying neutrinos and antineutrinos based on topological features
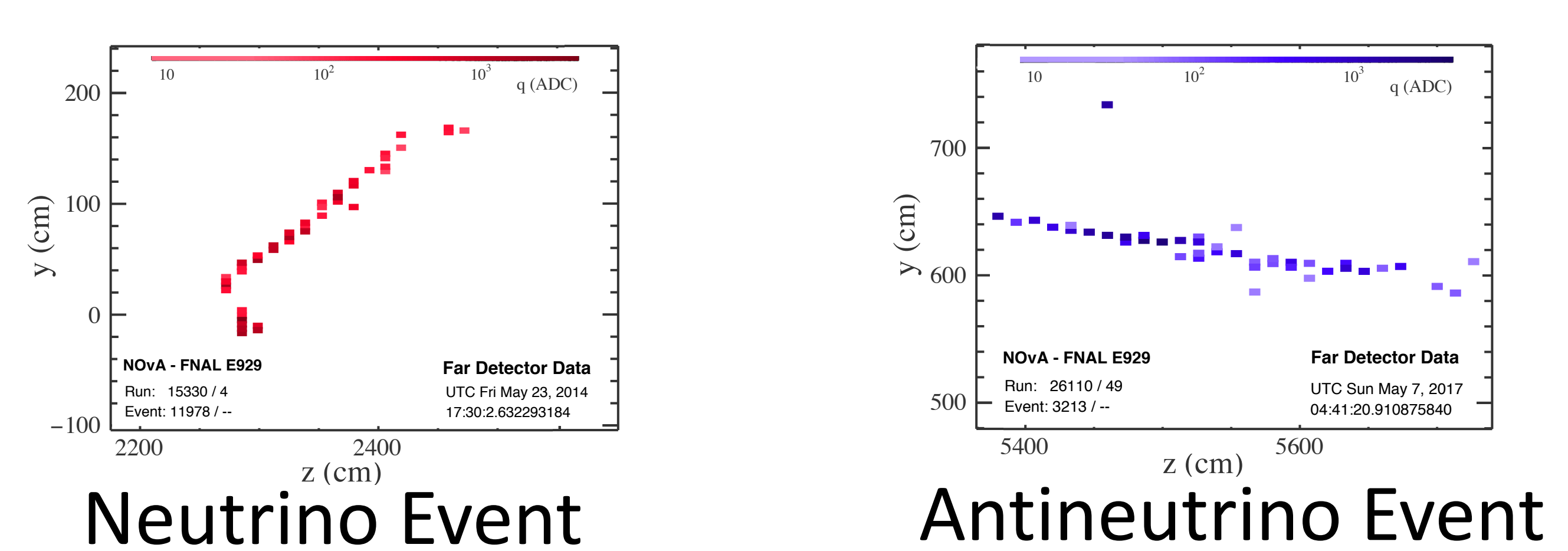

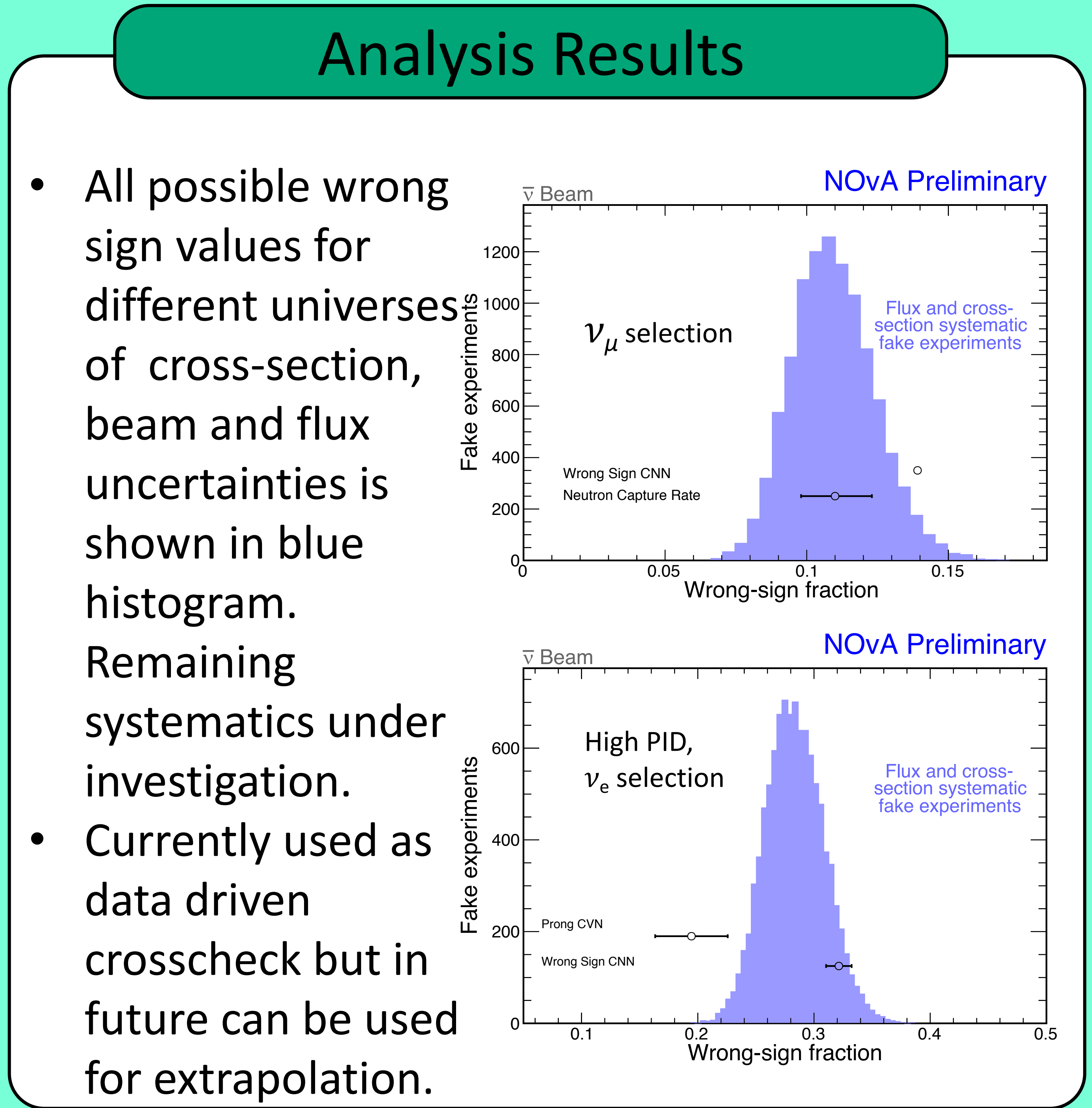

